\title{
Prevalencia de lateralidad y lenguaje receptivo en niños de 5 y 6 años del municipio de Corregidora Querétaro, México
}

\author{
Dra. Nadia Edith García Medina \\ Docente investigadora de la Facultad de Enfermería, \\ Universidad Autónoma de Querétaro, México \\ Yatziri Airaly Iñiguez Gasca \\ Alejandra Ugalde Hurtado \\ Estudiante de la Licenciatura en Fisioterapia, Facultad de Enfermería, \\ Universidad Autónoma de Querétaro, México \\ L. Ft. Karina González Zúñiga \\ Docente investigadora de la Facultad de Enfermería, \\ Universidad Autónoma de Querétaro, México \\ MC. Ángel Salvador Xeque Martínez \\ Docente investigador Facultad de Enfermería, \\ Universidad Autónoma de Querétaro, México
}

Doi:10.19044/esj.2021.v17n14p1

Submitted: 30 January 2021

Accepted: 08 March 2021

Published: 30 April 2021
Copyright 2021 Author(s)

Under Creative Commons BY-NC-ND

4.0 OPEN ACCESS

Cite As:

García Medina N.E., Iñiguez Gasca Y.A., Ugalde Hurtado A., González Zúñiga K. \& Xeque Martínez A.S. (2021). Prevalencia de lateralidad y lenguaje receptivo en niños de 5 y 6 años del municipio de Corregidora Querétaro, México. European Scientific Journal, ESJ, 17(14), 1. https://doi.org/10.19044/esj.2021.v17n14p1

\section{Resumen}

Introducción: La lateralidad es la preferencia del uso de una mitad del cuerpo sobre la otra, y toma en cuenta la dominancia de mano, ojo, oído y pie. Una lateralidad definida refiere la correcta comunicación entre los hemisferios cerebrales; la falta de consolidación de afirmación de la misma, se relaciona con trastornos en el aprendizaje del lenguaje y la lectoescritura. El test de Harris identifica la lateralidad en los niños y permite crear estrategias didácticas para lograr una afirmación en el niño, mientras que el test de Peabody da a conocer el nivel de lenguaje receptivo a partir de los 2 años. Objetivo: Determinar la prevalencia de lateralidad y lenguaje receptivo en niños de 5 y 6 años. Método: Estudio cuantitativo, transversal, descriptivo y 
observacional, realizado en niños de preescolar y primaria del municipio de Corregidora, Querétaro, México. A 229 estudiantes se les aplicó el Test de Harris y Peabody. Resultados: Mediante estadística descriptiva, se obtuvo que el $52 \%$ son preescolares, y el $48 \%$ de primero de primaria, siendo $53.3 \%$ niños y $46.7 \%$ niñas. Donde el $95.6 \%$ tiene lateralidad mal afirmada, mientras un $4.4 \%$ fueron diestros, además no hubo estudiantes con lateralidad cruzada o zurdos. El nivel de lenguaje receptivo en preescolares fue de alto a muy alto en un $56.1 \%$ de la población, mientras que los niveles de bajo a muy bajo solo fue el $43.8 \%$, mientras que en primaria el lenguaje receptivo mostró que el $66.6 \%$ de los estudiantes tienen niveles de alto a muy alto y solo un $32.4 \%$ de bajo a moderadamente bajo. Conclusión: Se muestra una falta de consolidación de la lateralidad debido a que un alto porcentaje de estudiantes manifiesta lateralidad mal afirmada, mientras que el lenguaje receptivo se adquiere adecuadamente con respecto a la edad.

Palabras claves: Lateralidad, lenguaje receptivo, trastorno de aprendizaje

\section{Prevalence of Laterality and Receptive Language in Children Aged 5 and 6 from the Municipality of Corregidora Querétaro, México}

\section{Dra. Nadia Edith García Medina}

Docente investigadora de la Facultad de Enfermería, Universidad Autónoma de Querétaro, México

Yatziri Airaly Iñiguez Gasca

Alejandra Ugalde Hurtado

Estudiante de la Licenciatura en Fisioterapia, Facultad de Enfermería,

Universidad Autónoma de Querétaro, México

\section{Ft. Karina González Zúñiga}

Docente investigadora de la Facultad de Enfermería,

Universidad Autónoma de Querétaro, México

MC. Ángel Salvador Xeque Martínez

Docente investigador Facultad de Enfermería,

Universidad Autónoma de Querétaro, México

\section{Abstract}

Introduction: Laterality is the preference of using one half of the body over the other, and takes into account the dominance of hand, eye, ear and foot. A defined laterality refers to the correct communication between the brain hemispheres; the lack of consolidation of affirmation of itself, is related 
to disorders in language learning and literacy skills. The Harris test identifies laterality in children and allows them to create teaching strategies to achieve an affirmation in the child, while the Peabody test shows the level of receptive language from the age of 2. Objective: To determine the prevalence of laterality and receptive language in 5 and 6 year old children. Method: Quantitative, transversal, descriptive and observational study, carried out with kindergarten and elementary children of the municipality of Corregidora, Querétaro, México. The Harris and Peabody tests were applied to 229 students. Results: Through descriptive statistics, $52 \%$ were preschoolers, and $48 \%$ were from first grade, $53.3 \%$ are boys and $46.7 \%$ girls. The $95.6 \%$ has poorly stated laterality, and $4.4 \%$ were right-handed, besides there weren't students with cross-laterality or left-handedness. The level of receptive language in preschoolers was from high to higher in $56.1 \%$ of the population, while low to lower levels were only $43.8 \%$, while in elementary the receptive language showed that $66.6 \%$ of students have levels from high to higher and only $32.4 \%$ from low to moderately low level. Conclusion: A lack of laterality consolidation is shown because a high percentage of students demonstrate poorly asserted laterality, while receptive language is properly acquired within the age.

Keywords: Laterality, receptive language, learning disorder

\section{Introduction}

La lateralidad es el predominio funcional de un lado del cuerpo sobre el otro, se manifiesta en la utilización de mano, pie, ojo y oído. La lateralidad corporal permite al niño relacionarse con su entorno y la afirmación de lateralidad tiene estrecha relación con la adquisición de habilidades cognitivas motoras, de lenguaje, matemáticas y creatividad. Dicha habilidad es una capacidad sensoriomotora y "sirve como un telar sobre el cual las fibras del lenguaje, la función ejecutiva, espacial y numérica, todas se entrelazan para crear el tejido de la cognición" (González C. L. R, 2018), indispensables para el desenvolvimiento académico y el éxito en este.

En México el logro educativo representa un enorme reto para aspirar al cumplimiento del derecho a la educación de calidad con equidad (INEE, 2009). Por ello se considera trascendental el incorporar la evaluación e identificación de la lateralidad como parte del diagnóstico en las dificultades en el lenguaje comprensivo, ya que de lo contrario puede impactar cuando el alumno está en escolaridad superior y aumentar el abandono académico (Van Dijk, 2012). Existe una clara relación entre la lateralidad mal afirmada y la presencia de niveles bajos en inteligencia lingüística, viso- espacial y kinestésico corporal (Casado, 2015). El abandono escolar es un problema de carácter público relacionado con el desenvolvimiento académico por lo que 
afectará la entrada del personal laboral, accesibilidad a educación superior, pobreza y exclusión social (García, 2016).

Las dificultades de lateralización pueden obstaculizar el aprendizaje de la lectura y la escritura, ya que previo a ello se requiere de la adquisición del lenguaje receptivo y expresivo; esto es una condicionante porque las letras se diferencian por su orientación lateral por ejemplo d y la b (Cuetos et al, 2015; Rodríguez et al, 2017; Fiuza \& Fernández, 2014). Es por ello, que es importante que los niños vayan teniendo un adecuado lenguaje receptivo, ya que este permite el desarrollo de las funciones necesarias para que pueda leer y escribir, cuando esto no sucede de acuerdo a la edad cronológica una de las causas es por un déficit en los procesos de la lateralidad.

El estado de Querétaro carece de este tipo de estudios por lo que el presente trabajo busca conocer cómo la lateralidad impacta en la adquisición del vocabulario comprensivo que existe a nivel preescolar en una escuela particular y en una pública perteneciente a la Unidad de Servicios para la Educación Básica en el Estado de Querétaro (USEBEQ).

Se ha descrito que en algunos países donde se ha presentado un porcentaje alto de deserción escolar no alcanzan un nivel académico medio superior o superior; en gran parte esto se vuelve más preocupante cuando un alumno se encuentra por concluir la educación básica, cuando debería de tenerse en cuenta que los primeros seis años de vida son esenciales para construir las bases del desarrollo y del aprendizaje. Por ello, se considera trascendental la participación del fisioterapeuta en la educación desde los niveles básicos, debido a que puede identificar la consolidación o alteración de lateralidad en el niño desde edades tempranas y junto los psicomotricista capacitados, implementar planes educativos donde a través del juego recreativo favorezcan la afirmación de la lateralidad en edades entre los 5 y 6 años (Mocha Bonilla, 2018; Mayolas, 2010). La lateralidad se distingue en tres fases, y depende del desarrollo del niño y su edad. La fase de indiferenciación, que ocurre desde el nacimiento hasta los 2 años, que se caracteriza porque, los niños realizan movimientos bilaterales en los que buscan principalmente descubrir su cuerpo y las partes que lo componen (manos, pies, cabeza, entre otros). Luego entre los dos y los cinco años, se presenta la fase de alternancia en la que los niños utilizan las dos manos y pies explorando con una y con otra mano o pie, el entorno y el ambiente que los rodea; es decir, que en esta fase los niños utilizan los dos pies y las dos manos, para la realización de sus actividades exploratorias en forma independiente. Para que luego, entre los 5 y los 6 años en la tercera fase, conocida como automatización, los niños logren tener una noción real de izquierda o derecha, logrando utilizar un lado dominante de su cuerpo. (Duarte, \& Pérez, 2020). La Prueba de Harris o de lateralidad (Harris Test of Lateral Dominance, 1957). Es una herramienta para determinar el tipo de predominio lateral de cada 
segmento corporal, conformado por 26 ítems divididos en cuatro apartados (10 actividades para mano y pie; 3 para ojo y oído) para valorar cómo se ha desarrollado este aspecto de la psicomotricidad.

Se considera importante conjuntar una valoración de lateralidad por medio de la prueba de Harris (1998) y el lenguaje receptivo por medio de la prueba de Peabody (Dunn, 2010). Ambas pruebas deberían se aplican en edades preescolares por parte de fisioterapeutas, docentes, pediatras y todo un equipo multidisciplinar de la salud del menor para dar un seguimiento adecuado, de acuerdo al desarrollo psicomotor, y así con ello detectar las dificultades motrices o de lenguaje en los primeros seis años de vida, y prevenir la afectación del aprendizaje en procesos como lo es la lectoescritura, trastornos de la coordinación, lateralidad cruzada, déficit vestibular, etc.

\section{Material y métodos}

Tipo de estudio cuantitativo, transversal, descriptivo y observacional, realizado en niños de preescolar y primaria del municipio de Corregidora, Querétaro, México. Se aplicó estadística descriptiva para resumir los datos, y las variables cualitativas se analizaron mediante distribución de frecuencias absolutas y relativas expresadas en porcentaje y frecuencia. La muestra estudiada se obtuvo de forma probabilística aplicando la fórmula de estimación de una proporción para poblaciones finitas; sustituyendo los valores en la formula correspondiente se obtuvo una muestra de 146 estudiantes para aplicar el instrumento, sin embargo la población final analizada fue de 229 estudiantes.

$$
n=\frac{Z_{\alpha / 2}^{2} p q N}{(N-1) E^{2}+Z_{\alpha / 2}^{2} p q}
$$

Donde:

Población ( $\mathrm{N}=236)$

Error 5\% $(\mathrm{E}=0.05)$

Éxito $50 \%(\mathrm{p}=0.5)$

Fracaso $50 \%(\mathrm{q}=0.5)$

Nivel de confianza de $95 \% \rightarrow\left(Z_{\alpha / 2}^{2}=1.96\right)$

\section{Participantes}

Participaron un total de 229 niños con un desarrollo psicomotor normativo y escolarizados de escuelas pertenecientes al Municipio de Corregidora del Estado de Querétaro. Tras la autorización del consentimiento informado de tutores y el asentimiento informado del menor, se aplicaron dos pruebas; Peabody III para conocer el nivel de lenguaje receptivo por medio de vocabulario en imágenes, y la prueba de Harris para identificar la dominancia 
lateral de cuatro segmentos: extremidad superior, extremidad inferior, ojo y oído. Para la selección de la muestra se tuvo en cuenta que los niños fueran saludables, que no presentaran antecedentes de alteraciones neurológicas, déficit intelectual, sensorial, motor o psiquiátrico que pueda comprometer su desempeño en la tarea, a través de la contestación de una historia clínica, con edades de entre los 5 y 6 años. Los estudiantes de 5 años conforman el grupo de preescolar y los de 6 años el grupo de primaria. Dos fisioterapeutas y dos psicomotricistas certificadas y capacitadas, aplicaron la prueba de Peabody por imágenes y la prueba de Harris sin interferir con las actividades propias de las escuelas. Se aplicaron los dos test de forma individual en un tiempo aproximado de 25 minutos.

\section{Evaluación de la lateralidad}

Se aplicó la prueba de Harris (Test de Albert J. Harris), el material a utilizar es de fácil adquisición y manipulación, las tareas a realizar son sencillas, y no son totalmente desconocidas para el niño, las pruebas son de fácil observación, y la tarea se realiza de forma espontánea. Por tal razón es una herramienta para determinar el tipo de predominio lateral de cada segmento corporal, conformado por 26 ítems divididos en cuatro apartados (10 actividades para mano y pie; 3 para ojo y oído) para valorar cómo se ha desarrollado la psicomotricidad. El material requerido es una hoja de registro por niño, una pelota chica y una mediana, lápiz, sacapuntas, pañuelos desechables, tijeras, hojas recicladas, cepillo de dientes, peine, plastilina, cuchillo (plástico), visor (cartón de 15 x $25 \mathrm{~cm}$ con un orificio de $0.5 \mathrm{~cm}$ de diámetro, telescopio y caleidoscopio; los resultados que arroja son los tipos de dominancia. La evaluación consta de dos fases; Fase 1, evaluar cada segmento corporal por separado y bajo la siguiente codificación según la prueba.

Preferencia de mano y pie (D: si realiza las 10 pruebas con la mano o pie derecho; I: si realiza las 10 pruebas con la mano o pie izquierdo; d: 7, 8 o 9 pruebas hechas con la mano o pie derecho; i: 7,8 o 9 pruebas hechas con la mano o pie izquierdo y x: todos los demás casos). Preferencia de ojo y oído (D: si utiliza el derecho en las tres pruebas; I: si utiliza el izquierdo en las tres pruebas, d: si utiliza el lado derecho en 2 de las 3, i: Si utiliza el lado izquierdo en 2 de las 3 pruebas y x: todos los demás casos). Fase 2, interpretación conjunta de la preferencia de los segmentos corporales bajo la siguiente interpretación: Diestro: Todas las actividades las realiza con hemicuerpo derecho (DDDD); Zurdo: Todas las actividades las realiza con hemicuerpo izquierdo (I I I I); Lateralidad cruzada: Cuando la mano dominante y el ojo dominante se encuentran en lados opuestos (DIDI) y Lateralidad mal afirmada: No muestra preferencia consistente por una mano o superioridad de la misma (ddDd y todas las demás variantes). El tiempo de aplicación es de 10 a 15 minutos aproximadamente. 


\section{Evaluación del lenguaje comprensivo}

La prueba de vocabulario en imágenes de Peabody III, determina el nivel de lenguaje receptivo, y se utiliza para la evaluación antes de la alfabetización (adquisición del vocabulario como indicador del desarrollo lingüístico), permite realizar una detección rápida de las dificultades de la aptitud verbal y no es necesario que el sujeto evaluado tenga conocimiento de la lectura; está compuesta por 7 conjuntos de 84 elementos, en donde al niño se le muestra una lámina con cuatro imágenes y tiene que señalar aquella a la que corresponda la palabra pronunciada por el evaluador. Se considera una medida estandarizada del lenguaje receptivo, porque es una de las escasas pruebas que existen en español para medir aspectos lingüísticos a edades tempranas. Aunque solo mide la compresión por vía auditiva, su extendido uso le confiere un valor referencial destacable; es considerada como una de las pruebas más validadas para conocer el nivel de habilidades lingüísticas. El material utilizado fueron dos sillas, una mesa, una hoja de evaluación por niño y cuadernillo de imágenes; los resultados obtenidos se clasifican en dos tipos: puntuaciones desviación y puntuaciones desarrollo. Las puntuaciones del coeficiente intelectual $(\mathrm{CI})($ media $=100 ; \mathrm{dt}=15)$, los percentiles y los eneatipo (media=5; $\mathrm{Dt}=2$ ) estos informan sobre lo desviado que está el resultado del niño, si es normalizado o no para la edad. La puntuación de desarrollo es la edad equivalente, la cual indica la edad en años y meses el rendimiento del individuo de acuerdo a su edad cronológica. El tiempo de aplicación es de aproximadamente 12 minutos. Este test permite clasificar el nivel de lenguaje receptivo en: muy bajo, moderadamente bajo, bajo, alto, moderadamente alto y muy alto, tomando en cuenta las puntuaciones del CI, eneatipo y percentiles tal y como se muestra en la figura 1. 


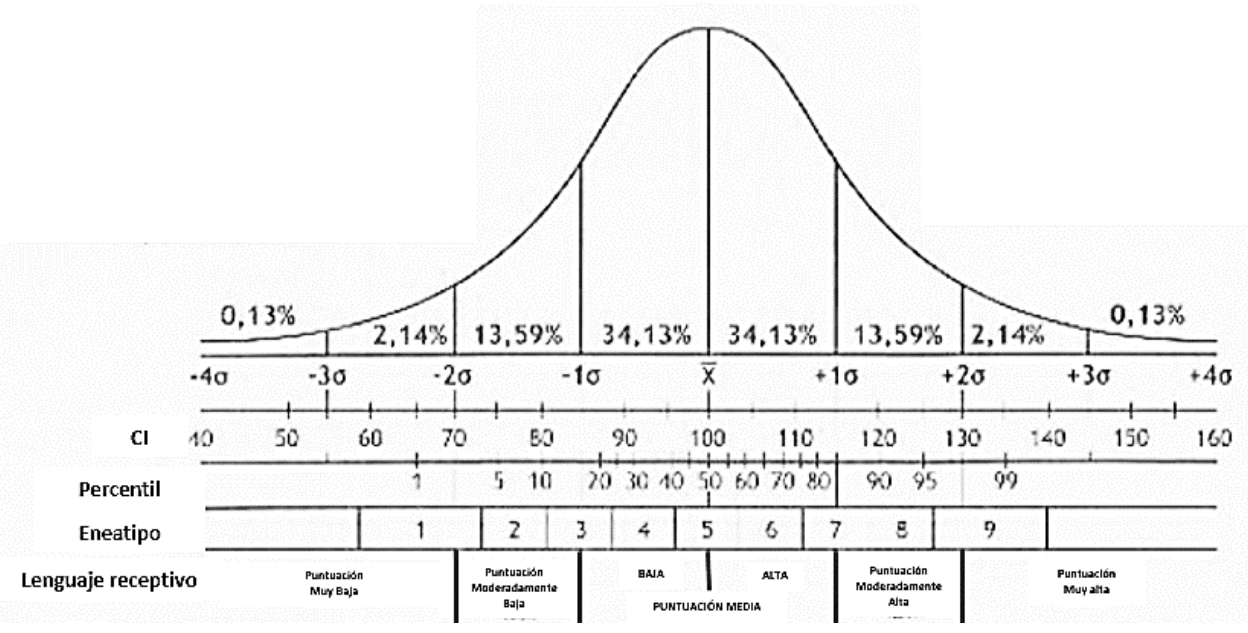

Figura 1. Curva normal de la prueba de Peabody III; según el coeficiente intelectual (CI), el percentil y eneatipo obtenido por cada sujeto evaluado, se traza una línea recta en vertical que corte con las otras escalas, para conocer el nivel de lenguaje receptivo (Dunn, 2010).

\section{Resultados}

La población en estudio estuvo integrada por un total de 229 estudiantes, de los cuales el 52\% ( $\mathrm{n}=119)$ correspondía a estudiantes de preescolar, mientras que un $48 \%(\mathrm{n}=110)$ a estudiantes de primaria. El $53.3 \%$ niños y $46.7 \%$ niñas. Con respecto a la lateralidad $95.6 \%(n=219)$ estaba mal afirmada, mientras que un $4.4 \%(\mathrm{n}=10)$ eran diestros, ningún estudiante mostró lateralidad cruzada o ser zurdos.

Del $52 \%$ de la población de preescolares, la edad de los estudiantes oscila entre los 5 y 5 años 10 meses, siendo el $53.8 \%$ niños (64/119) y el $46.2 \%$ niñas (55/119). No se obtuvieron estudiantes zurdos o con lateralidad cruzada, pero el $95.8 \%(114 / 119)$ tenía lateralidad mal afirmada mientras que sólo el $4.2 \%(5 / 119)$ ya está definida como diestros. En este grupo de preescolares aquellos estudiantes que tuvieron lateralidad mal afirmada además mostraron tener un nivel de lenguaje receptivo según Peabody de alto a muy alto un $56.1 \%$ (64/114) de los estudiantes, mientras que el 43.8\% (50/114) tuvieron niveles de lenguaje receptivo de bajo a muy bajo. Con respecto a los que tuvieron lateralidad definida como diestra, el $80 \%$ de los estudiantes (4/5) tienen un lenguaje alto, y el 20\% (1/5) únicamente bajo.

Respecto al grupo de estudiantes de primero de primaria $48 \%$, la edad de los estudiantes oscila entre los 6 y 7 años 1 mes, siendo el $52.7 \%$ niños (58/110) y el $47.3 \%$ niñas (52/110). No se obtuvieron estudiantes zurdos o con lateralidad cruzada, pero el $95.5 \%$ (105/110) tenía lateralidad mal afirmada mientras que solo el 4.5\% (5/110) ya está definida como diestra. En este grupo de primero de primaria, los estudiantes que tuvieron lateralidad mal afirmada 
además mostraron tener un nivel de lenguaje receptivo según Peabody de alto a muy alto un $66.6 \%(70 / 105)$ de los estudiantes, mientras que el $32.4 \%$ (35/105) tuvieron niveles de lenguaje receptivo de bajo a muy bajo. Con respecto a los que tuvieron lateralidad definida como diestra, el $60 \%$ de los estudiantes (3/5) tienen un lenguaje alto, y el $40 \%$ (2/5) de bajo a moderadamente bajo. Resultados mostrados en la tabla 1.

TIPO DE LATERALIDAD

\begin{tabular}{|c|c|c|c|c|c|c|c|c|}
\hline \multirow{4}{*}{$\begin{array}{l}\text { NIVEL DE } \\
\text { LENGUAJE }\end{array}$} & \multirow{2}{*}{\multicolumn{4}{|c|}{ PREESCOLAR }} & \multirow{2}{*}{\multicolumn{4}{|c|}{ PRIMARIA }} \\
\hline & & & & & & & & \\
\hline & \multicolumn{2}{|c|}{ Diestro } & \multicolumn{2}{|c|}{ Mal afirmada } & \multicolumn{2}{|c|}{ Diestro } & \multicolumn{2}{|c|}{ Mal afirmada } \\
\hline & \multirow[t]{2}{*}{$\mathbf{n}$} & \multirow[t]{2}{*}{$\%$} & \multirow[t]{2}{*}{$\mathbf{n}$} & \multirow[t]{2}{*}{$\%$} & \multirow[t]{2}{*}{$\mathbf{n}$} & \multirow[t]{2}{*}{$\%$} & \multirow[t]{2}{*}{$\mathbf{n}$} & \multirow[t]{2}{*}{$\%$} \\
\hline RECEPTIVO & & & & & & & & \\
\hline Muy Alto & 0 & 0 & 1 & 0.8 & 0 & 0 & 0 & 0 \\
\hline Moderadamente Alto & 2 & 40 & 13 & 11.4 & 0 & 0 & 16 & 15.2 \\
\hline Alto & 2 & 40 & 50 & 43.9 & 3 & 60 & 54 & $\mathbf{5 1 . 4}$ \\
\hline Bajo & 1 & 20 & 38 & 33.3 & 1 & 20 & 28 & 26.7 \\
\hline Moderadamente Bajo & 0 & 0 & 8 & 7 & 1 & 20 & 6 & 5.7 \\
\hline Muy Bajo & 0 & 0 & 4 & 3.5 & 0 & 0 & 1 & 0.9 \\
\hline Total & 5 & 100 & 114 & 100 & 5 & 100 & 105 & 100 \\
\hline
\end{tabular}

Tabla 1. Tipo de lateralidad y lenguaje obtenido en estudiantes de preescolar y primero de primaria de escuelas privadas y públicas

\section{Discusión}

Según los resultados obtenidos tanto en niños de preescolar como de primero de primaria, hay un alto porcentaje que tienen lateralidad mal afirmada, lo que pudiera provocar bajo desempeño escolar o de aprendizaje, algunos autores han propuesto que entre más afianzada y fuerte sea la lateralidad (diestra o zurda), mejor será la capacidad cognitiva (Barrero et al., 2016), además el lenguaje y la comprensión del mismo favorece los mecanismos para el aprendizaje. En este sentido, los estudiantes de preescolar tienen un nivel de alto a muy alto de lenguaje receptivo, pero con una lateralidad mal afirmada, esto indica que el lenguaje se está adquiriendo y desarrollando en tiempo, y que su lateralidad puede alcanzar la consolidación para evitar aspectos fonológicos, semánticos, sintácticos y pragmáticos, y un lenguaje sin dificultades de comprensión o articulación (Morales et al., 2013; Moreno, 2013). Por otra parte se debe reforzar la psicomotricidad dando mayor importancia a trabajar lateralidad visual y auditiva, para propiciar que haya un alcance del buen neurodesarrollo, tal y como menciona Cabañas (2008) “...los problemas psicomotrices, pueden ser ocasionados por situaciones de inmadurez, disfunción de los patrones motrices, sensoriales, $o$ de desarrollo que el cerebro utiliza a través de redes neuronales para asociar $e$ integrar la información y seleccionarla". Se conoce que los patrones motrices como es el caso de la lateralidad, han relacionado a la lateralidad mal afirmada o cruzada con problemas de aprendizaje en los primeros años de escolaridad (Mayolas et al. ,2010; Castejón, 2013), y las dificultades de 
lateralización pueden obstaculizar el aprendizaje de la lectura y la escritura, ya que previo a ello se requiere de la adquisición del lenguaje receptivo y expresivo; esto es una condicionante porque las letras se diferencian por su orientación lateral por ejemplo "d" y la "b" (Cuetos et al, 2015; Rodríguez et al, 2017; Fiuza \& Fernández, 2014).

En los estudiantes de primero de primaria, el $95.5 \%$ aún no consolidan su lateralidad, y sus edades ( 6 a 7.1 años) corresponden a tener una lateralidad definida. Algunos investigadores como Vela et al. (2016) y Korzeniowski (2010), concuerdan que a partir de los 4 años los niños deben ya mostrar la preferencia de ojo, oído, mano y pie, siendo estos elementos la base para el aprendizaje. Entre los 5 y 6 años, es un momento de la vida crítico y clave, puesto que es la etapa en donde se preparan los niños para el ingreso a nivel escolar; es importante resaltar que la detección de las probables limitaciones funcionales, que les impiden desarrollar las competencias básicas para ingresar a la escuela primaria, puedan ser atendidas oportunamente antes de alcanzar el nivel medio y superior de su escolaridad.

Con respecto al género, en ambos grupos, se observó un mayor número de estudiantes con género masculino y lateralidad mal afirmada, este resultado concuerda con lo ya reportado por Ileana y colaboradores (2013), mientras en ambos grupos se observa un nivel de lenguaje receptivo adecuado. Está descrito por algunos autores que en los primeros 6 años de vida es fundamental el desarrollo del lenguaje puesto que se deben consolidar aspectos fonológicos, semánticos, sintácticos y pragmáticos que le permitirán al niño desarrollar un lenguaje sin dificultades de comprensión o articulación (Morales et al., 2013; Moreno, 2013). Autores como Lévano (2018) mencionan que el vocabulario comprensivo aumenta en función de la edad, siendo un proceso continuo en niños a partir de los 2 años, por ello nuestros resultados muestran que desde los estudiantes de preescolar a partir de los 5 años y hasta los 7 años un mes de primaria van de alto a muy alto en el lenguaje receptivo según Peabody, pero no es el caso con la consolidación de la lateralidad.

Con ello, la lateralidad, es un tema importante para maestros de preescolar y primaria dado que parece clara la existencia de una relación entre la lateralización hemisférica y la lateralidad corporal, siendo motivo de estudio a la hora de determinar las causas que producen trastornos en el aprendizaje (Pi \& Masi, 2015), esto quiere decir que cada uno de los segmentos corporales funciona como referencia para desarrollar la lateralidad y a su vez complementa la maduración del lenguaje y lectoescritura, de lo contrario puede existir un impacto negativo tanto en sus relaciones sociales como en el logro de sus habilidades cognitivas como la dislexia, disgrafía y disortografía (Luque, 2018); el dominio de la lateralidad contribuye a integrar aspectos de 
direccionalidad y equilibrio, esto le ayudará en un futuro a ubicarse en una hoja en blanco o en un texto.

La lateralidad está directamente implicada en el rendimiento escolar, principalmente en los procesos relacionados con el lenguaje, la lectoescritura o las matemáticas (Timoneda et al., 2013) y por tal razón, es necesario que los fisioterapeutas o psicomotricistas que laboral con instituciones de nivel escolar básico sigan evaluando e implementando actividades recreativas en los preescolares, y reforzar sus clase de psicomotricidad y /o educación física con la finalidad de que consoliden su lateralidad, y evitar alteraciones de aprendizaje en los primeros niveles de primaria.

\section{Conclusión}

Según los resultados aquí mostrados el lenguaje receptivo se adquiere adecuadamente con respecto a la edad, mientras que en la lateralidad se muestra una falta de consolidación de la lateralidad debido a que un alto porcentaje de estudiantes manifiesta lateralidad mal afirmada, y esto puede repercutir en aprendizaje de lectoescritura y aprendizaje en primaria. Es necesario seguir trabajando con los estudiantes de preescolar y fomentar el movimiento en para reforzar la comunicación interhemisférica y por tanto favorecer que consoliden su lateralidad para mejorar su aprendizaje y desarrollo cognitivo.

\section{Agradecimientos}

Agradecemos el apoyo al Colegio Viktor Frankl en especial a las licenciadas Gloria Paula Castillo Portela quien es Subdirectora de psicopedagogía y Seltone Castañeda Yamin Subdirectora Académica, al Preescolar "El Movi" en particular a la Directora Académica la Lic. Gemma América González Estrada y a la Lic. Julia Andrea Martínez Meneses. Todas ellas autoridades importantes en la logística de intervención con padres y con los niños.

A los alumnos de prácticas pediátricas Chama Avilés Daniel Sebastián, Loeza Narváez Wendy Alejandra, Lozada Paquini David, Martínez González Abigail, Bárcenas Cervantes Areli, y García Hernández Rafael, por su valioso apoyo en la logística del estudio.

\section{Fuentes de financiamiento}

La presente investigación no ha recibido ninguna beca específica de agencias de los sectores público, comercial o sin ánimo de lucro.

\section{Conflicto de interés}

Ninguno 


\section{References:}

1. Barrero, M., Vergara-Moragues, E. \& Martín-Lobo, P (2016). Avances neuropsicológicos para el aprendizaje matemático en educación infantil: la importancia de la lateralidad y los patrones básicos del movimiento. Edma 0-6: Educación Matemática en la Infancia, [S.1.], v. 4, n. 2, p. 22-31, oct. 2020. ISSN 2254-8351.

2. Cabañas, F. (2008). Lesión cerebral en el niño prematuro. Asociación Española de Pediatría (2002). Protocolos diagnósticos y terapéuticos de neonatología en pediatría.

3. Casado, Y. (2015). Inteligencias múltiples, creatividad y lateralidad, nuevos retos en metodologías docentes enfocadas a la innovación educativa. REIDOCREA, 4(34), 343- 358.

4. Castejón, J. L. \& Navas, L. (2013) Dificultades y trastornos del aprendizaje y del desarrollo en infantil y primaria. Editorial Club Universitario.

5. Cuetos, F., Suárez-Coalla, P., Molina, M. I., \& Llenderrozas, M. C. (2015). Test para la detección temprana de las dificultades en el aprendizaje de la lectura y escritura. Pediatría Atención Primaria, 17(66), e99-e107. https://dx.doi.org/10.4321/S113976322015000300002.

6. Duarte, F. J. \& Pérez, N. B. (2020). Identificar la lateralidad en niños de 2 a 5 años del instituto de recreación y deportes de tunja (IRDET) aplicando el test de Harris. Revista digital: Actividad Física y Deporte. 6(2):118-144. https://doi.org/10.31910/rdafd.v6.n2.2020.1572.

7. Dunn, L. M. \& Arribas, D. (2010). Peabody. Test de vocabulario en imágenes.

8. Ferradas, C. (2015). Evaluación de la lateralidad mediante el test de harris en niños de 3 y 6 años. Valladolit.

9. Fiuza, M. \& Fernández, P. (2014). Dificultades de aprendizaje y trastornos del desarrollo. Manual didáctico. Ediciones Pirámide. Madrid.

10. García, B. (2016). Indicadores de abandono escolar temprano: un marco para la reflexión sobre estrategias de mejora. Perfiles educativos, 38(154), 191-213.

11. Gonzalez, C. L. R., van Rootselaarn, N.A. \& Gibb, R. L. (2018). Sensorimotor lateralization scaffolds cognitive specialization. The Brain in Action Laboratory, Department of Kinesiology, University of Lethbridge. 238:405-433. https://doi.org/10.1016/bs.pbr.2018.06.011

12. Harris, A. J. (1957). Test de dominancia lateral.

13. Harris, A. J. (1998). Test de dominancia lateral.

14. Ileana, D. C., Álvarez, V., Gisela, I. D., Abadal, V., Elizabeth, D., Sánchez, G.,... Pérez, L. (2013). Factores biológicos asociados al 
retardo primario del lenguaje en niños menores de cinco años Biological factors associated to delayed primary language development in the under five years-old children, 85(4), 466-475.

15. INEE. (2009). El Derecho a la educación en México Informe 2009. 2020. Instituto Nacional para la Evaluación de la Educación.

16. Korzeniowski, C. (2010). Desarrollo evolutivo del funcionamiento ejecutivo y su relación con el aprendizaje escolar. Revista de Psicología. UCA. 2011. Vol. 7, No 13. ISSN 1669-2438.

17. Lévano Camones, K. R. (2018). Vocabulario comprensivo en niños de 2 a 6 años del centro de terapias. Tesis de licenciatura. Universidad Nacional Federico Villareal.

18. Luque C.M. (2018). Prevención de dificultades lectoras y escritoras en Educación Infantil. Revista Internacional de Apoyo a la Inclusión, Logopedia, Sociedad y Multiculturalidad 4(3), 56-65.

19. Mayolas, M.C., Villarroya, A. \& Reverter, J. (2010). Relación entre la lateralidad y los aprendizajes escolares. Apunts Educación Física y deportes, $121,3^{\circ}$ trimestre, 32-44.

20. Mocha, J. A., Coba M., \& Edison. (2018). Efectos de un programa de juegos recreativos en la definición de la lateralidad. Espacios, 39, 26.

21. Morales, P. T., Elizabeth, D., \& Ramos, G. (2013). Factores de riesgo perinatal, signos neurológicos blandos y lenguaje en edad preescolar, 12(3), 128-133.

22. Moreno-Flagge N. (2013). Trastornos del lenguaje. Diagnóstico y tratamiento. Rev. Neurol; 57 (Supl 1): S85-94.

23. Pi, C. M., \& Masi, R. (2015). Influencia de la edad y el género en los fenotipos y coeficientes de lateralidad en niños de 6 a 15 años, 11-18.

24. Rodríguez, T., Gómez, I., Prieto-Ayuso, A., \& Gil-Madrona, P. (2017). La educación psicomotriz en su contribución al desarrollo del lenguaje en niños que presentan necesidades específicas de apoyo educativo. Revista de Investigación en Logopedia, 7(1), 89-106. ISSN: 2174-5218.

25. Timoneda, C. Pérez, F., Mayoral, S. \& Serra, M. (2013). Diagnóstico de las dificultades de lectura y escritura y de la dislexia basado en la Teoría PASS de la inteligencia utilizando la batería DN-CAS. Origen cognitivo de la dislexia., Vol. 41, No 1, págs. 5-16. ISSN 0210-2773.

26. Van Dijk, S. (2012). La política pública para abatir el abandono escolar y las voces de los niños, sus tutores y sus maestros. Revista mexicana de investigación educativa, 17(52), 115-139.

27. Vela, M., Sánchez, A., \& Torres, J. (2016). Las capacidades perceptivo motoras en el preescolar. Una alternativa para su caracterización y desarrollo. Editorial REDIPE. Santiago de Cali, Valle del Cauca, Colombia. 